\title{
Is medical genetics neglecting epigenetics?
}

\author{
Arthur L. Beaudet, MD, Harland Sanders Award Recipient
}

Lifetime achievement means a lifetime of colleagues to thank. I would especially like to thank the many students and postdoctoral fellows who have worked with me over the years. It seems inevitable that junior scientists never receive the share of the credit that they deserve. I would also like to thank many colleagues both within and outside Baylor College of Medicine for invaluable contributions. I would also like to thank my family, especially my wife, Margie, for supporting or at least surviving my unbalanced lifestyle with excessive commitment to science and work at the expense of family.

I would also like to echo a comment made by Bruce Korf a few minutes ago emphasizing the scientific excitement in medical genetics, and our good fortune to participate at a time of unprecedented discovery in genetics and medicine. I remember thinking that some of my grandparents had the good fortune to live from the era of horse and buggy days to cars, airplanes, and landing on the moon. In terms of biology, I feel that I am having the good fortune to witness a similar explosion in scientific knowledge and biomedical research from delineation of the genetic code to recombinant DNA techniques-and all that they have made possible- to the ongoing completion of the Human Genome Project. I cannot imagine having lived through a more exciting time in biology, but the future is likely to prove even more productive than the present.

I titled these comments with a question: Is medical genetics neglecting epigenetics? My answer is yes, and I will try to tell you why. Others have discussed the relevance of epigenetics to disease, ${ }^{1,2}$ but the issue does not seem to have captured the imagination of clinical geneticists. On a personal note, I have always felt that my research career has lacked focus, although I have thoroughly enjoyed being engaged in diverse fields. Now for my waning, but hopefully still significant, years in research, I believe that I have found a focus, and it is epigenetics and genomic imprinting in medicine and human biology. My interest in genomic imprinting began when we encountered a teenage girl with cystic fibrosis and short stature secondary to maternal uniparental disomy (UPD) for chromosome $7 .^{3}$

Genomic imprinting is a part of the broader field of epigenetics which was described in a recent entertaining commentary as "the study of changes in gene function that are heritable and do not entail change in DNA sequence." I would suggest that "heritable" be changed to "stable and potentially heritable" to include the possibility that terminally differentiated

\footnotetext{
From the Department of Molecular and Human Genetics, Baylor College of Medicine, Houston, Texas.

Arthur L. Beaudet, MD, Department of Molecular and Human Genetics, Baylor College of Medicine, One Baylor Plaza, T619, Houston, TX 77030.
}

DOI: 10.1097/01.GIM.0000029041.28039.9F cells might stably and reversibly alter gene function without altering DNA sequence. Epigenetics involves numerous biochemical features including methylation of cytosine at CpG dinucleotides in DNA, acetylation of histones, methylation of histones, and other changes in chromatin. The definition does not imply that epigenetics includes all transcriptional control, because much of gene regulation is quite labile and subject to various forms of feedback. However, some intermediate forms of gene regulation might be difficult to categorize as to whether they are part of epigenetics or not. It seems likely that there is no sharp boundary between forms of gene regulation that are stable enough to merit inclusion as epigenetic and less stable forms of regulation. Epigenetics is not as neglected in all of biology as it is in medicine, and many scientists have emphasized its importance. In 1995, Robin Holliday, ${ }^{5}$ referring to methyl-cytosine, wrote, "there are not four bases in human DNA, but at least five, and very likely others." He went on to say that "organisms have many different epigenotypes in their cells and tissues." An epigenotype might be defined as the sum of the stable alterations of DNA and chromatin across a region. There is potential for an almost infinite number of different epigenotypes within the individual cells of our body, and these may be changing extensively with development, age, and other processes. The term epigenomics has been suggested to describe the enormously diverse information that is reflected in epigenotypes, and a European Human Epigenome Consortium has been initiated with a commercial component, Epigenomics GmbL. ${ }^{6}$ A similarly broad perspective is implied by the description of the histone code to include the chromatin changes that are superimposed on the DNA code. ${ }^{7}$

I would like to raise a few speculative questions about how epigenetics might be far more important in human biology and disease than is generally recognized. What is the role of epigenetics in the function and plasticity of human brain? Does learning change DNA methylation? How extensively are individual CNS cells using epigenetics to carry out their functions? What is the role of epigenetics in the recent primate evolution? Are the differences between mouse and human primarily epigenetic and not genetic? The analogy of the DNA genomic sequence to a text is drawn quite commonly. It may be reasonable to extend this analogy to suggest that epigenetics is the word processing font of the genetic code (Fig. 1), although the primary letters might not vary; the font might be large or small; Times Roman or Arial; italics, bold, or normal; uppercase or lowercase; underlined, shadowed, or embossed; superscript or subscript; even in strikethrough; and any color of the rainbow. Perhaps this can allow us to envision how the function of the genome can be modified very extensively without changing the primary sequence. The genotype can affect the phenotype only 


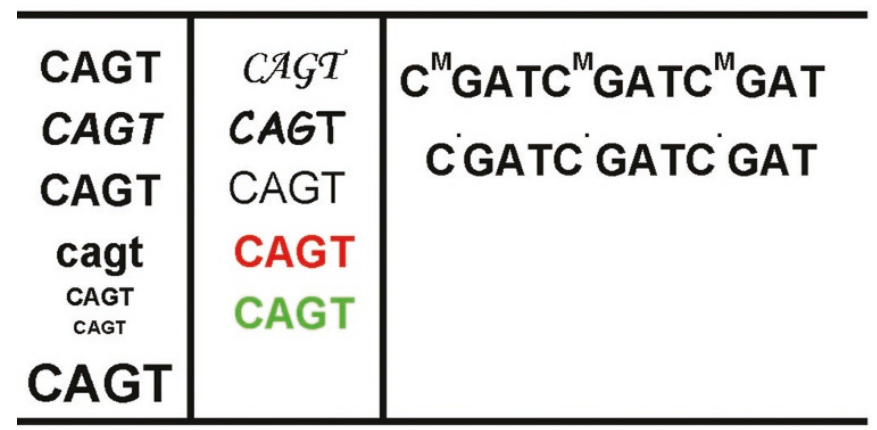

Fig. 1 Epigenetics as the font of the genetic alphabet of the genotype.

through the prism of the epigenotype (Fig. 2). The most obvious and decipherable form of epigenetic information is the methylation pattern at $\mathrm{CpG}$ dinucleotides. This can be measured using methylation-sensitive restriction enzymes or better using the bisulfite sequencing technique. ${ }^{8}$ We can easily imagine having the bisulfite sequence of the human epigenome, but there will be an almost infinite number of bisulfite sequences in different cells in a human organism. However daunting this complexity may be, we have no alternative but to begin to approach the task of understanding human epigenotypes.

I think it is interesting to contrast genetic disease and epigenetic disease, defining the former as an aberration in the nucleotide sequencing causing a disease phenotype and the latter as an aberration in epigenotype (stable/heritable change in gene expression) causing a disease phenotype in the absence of a nucleotide alteration. A straightforward example can be drawn from the Prader-Willi and Angelman syndromes. The majority of patients with these disorders have a deletion of chromosome 15q11-q13, and this is clearly a genetic disease by the above definitions (Fig. 3). In contrast, a smaller fraction of these patients have UPD, thus an epigenetic disease. There is no abnormality of the human genomic sequence in individuals with Prader-Willi syndrome or Angelman syndrome caused by

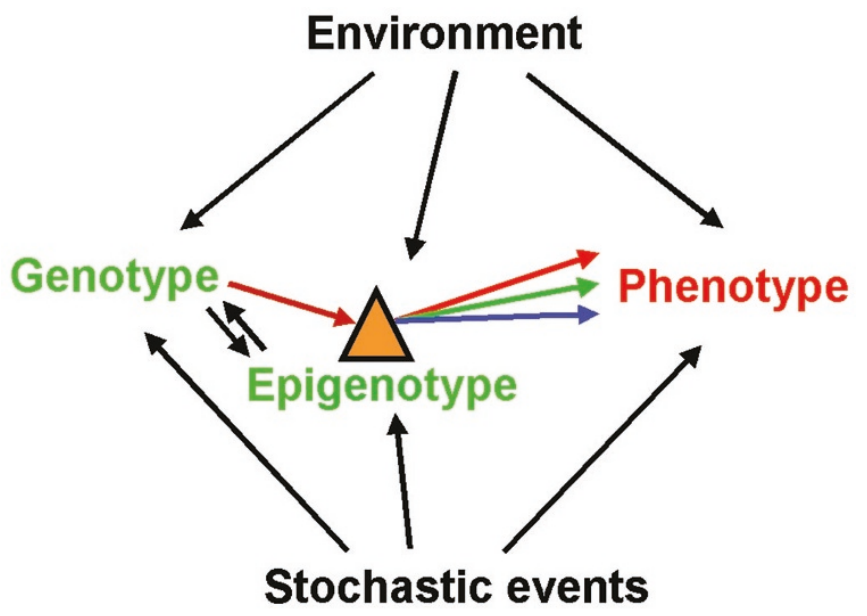

Fig. 2 The genotype affects the phenotype only through the prism of the epigenotype.

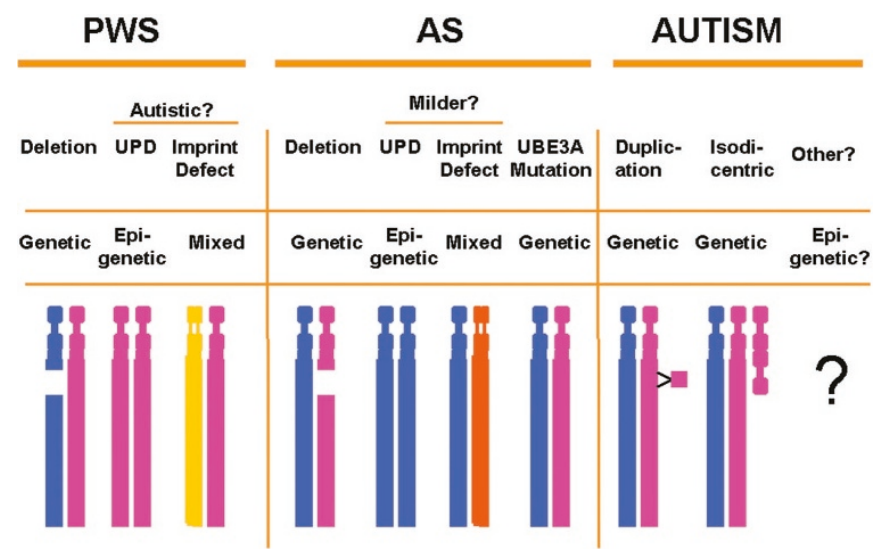

Fig. 3 Prader-Willi syndrome (PWS), Angelman syndrome (AS), and autism as genetic and epigenetic diseases of chromosome 15q11-q13. Blue represents paternal origin and paternal epigenotype; pink represents maternal origin and maternal epigenotype; yellow represents paternal origin but maternal epigenotype; orange represents maternal origin but paternal epigenotype.

UPD. Thus we see a distinct phenotype that can be caused by a genetic change in some of the patients and an epigenetic change in others. Upon reflection, it is obvious that all of the phenotypes consistently associated with one or another form of UPD represent epigenetic disease entities. It is interesting to speculate whether epigenetic contributions might be frustrating some genome-wide scans to identify genes contributing to one or another phenotype. Perhaps epigenetics is important in complex traits and as a modifier of what we generally think of as single-gene disorders. I think that it is particularly stimulating to think about the possibility that phenotypes such as schizophrenia, bipolar disorder, depression, or autism might be primarily epigenetic rather than genetic diseases. Others have pointed out the potential relevance of epigenetics to psychiatric illness. ${ }^{9}$ The etiology of a phenotype might represent a complex mixture of genetic, epigenetic, and other contributions. In the case of human cancer, there is a recent but greater awareness of the importance of epigenetics, and it would be relatively well accepted to propose that an individual colon cancer might involve a multistep process that would include stochastic mutations, environmentally induced mutations, stochastic epimutations, and environmentally induced epimutations. In addition, mechanisms such as viral contributions might be relevant for some forms of cancer. It is of interest to try to suggest examples of human disease phenotypes, other than cancer, in which a mixture of genetic and epigenetic factors causes the phenotype of an individual. As we have heard in the presentations about phacomatoses today, we can imagine that at least the tumor components of these disorders represent a mixture of genetic and epigenetic alteration. In thinking about the potential contributions of epigenetics to disease processes, it would seem that the epigenotype might be intrinsically less stable than the genotype, and therefore more susceptible to stochastic or environmentally induced alterations.

I would like to comment about autism and the growing awareness that chromosome $15 \mathrm{q} 11-\mathrm{q} 13$ is important in at 
least a small fraction of these patients. There are reports that maternal but not paternal interstitial duplications of this region cause a typical autism phenotype (Fig. 3 ). ${ }^{10}$ In addition, isodicentric or inverted duplicated chromosomes 15 are found in autism patients, and the extra chromosome is always of maternal origin if the phenotype is one of autism. ${ }^{11-13}$ These data strongly suggest that overexpression of a maternally expressed gene in 15q11-q13 causes autism in at least a few percent of cases, and I would suggest that UBE3A (the Angelman gene) is the best, but not the only, candidate gene in 15q11-q13 for contributing to the etiology of autism. Angelman syndrome and autism are behavioral opposites, with Angelman patients happy and interactive compared with the withdrawn behavior of autism. I would speculate that overexpression of UBE3A due to maternal or paternal defects may account for some larger fraction of autism than is currently appreciated. I believe it is even possible that a majority of autism might be caused by epigenetic defects in the expression of $U B E 3 A$, and these hypothetical epigenetic defects could be brain-specific, and not diagnosable using DNA from blood or other non-CNS tissues.

The twin data for autism are quite intriguing. The concordance for monozygotic (MZ) twins is reported to be $36 \%$ to $91 \%$, usually closer to $90 \% .{ }^{14}$ In contrast, the concordance for dizygotic (DZ) twins is often reported to be 0 and is presumably $<1 \%$ to $3 \%$. It is of interest to compare the expected MZ and $\mathrm{DZ}$ concordance for various genetic etiologies. One would expect the MZ concordance to be $100 \%$ for most single-gene and chromosomal disorders including cystic fibrosis, Huntington disease, neurofibromatosis, Down syndrome, and achondroplasia. The concordance in DZ twins is more variable. For an autosomal recessive disorder such as cystic fibrosis, the $\mathrm{DZ}$ concordance would be predicted to be $14 \%$ if one ascertained only twin pairs where at least one individual was affected with cystic fibrosis. In considering Huntington disease or inherited neurofibromatosis, the concordance for DZ twins would be predicted to be $33 \%$ if only twin pairs with at least one affected individual were ascertained. New mutation cases of achondroplasia and trisomy 21 have very low concordance rates for $\mathrm{DZ}$ twins, because they are caused by de novo defects affecting an individual germ cell, and DZ twins do not share common gametes. By analogy to genetic disorders, one can envision epigenetic disorders that might be semiheritable and others that might represent de novo events. If one imagines de novo epigenetic abnormalities arising in sperm or eggs, one might predict a concordance of $100 \%$ for MZ twins and some very low concordance for DZ twins. The interstitial maternal duplications and the isodicentric $15 \mathrm{q}$ abnormalities causing autism demonstrate the potential for both inherited and de novo genetic defects in a small fraction of cases, and it is tempting to wonder whether related epigenetic defects might represent a major cause of autism, by analogy to the genetic and epigenetic forms of Prader-Willi and Angelman syndromes (Fig. 3).
I would like to conclude by calling attention to some interesting data from a Dutch famine that occurred after World War II and to the potential relevance to folic acid supplementation in our diets. The famine in question occurred from October 1944 to May 1945, and the general characteristics of this unfortunate episode have been described. ${ }^{15,16}$ There was a 50\% drop in birth rate 9 months after the peak of the famine, perhaps related to changes in ovulatory cycle. There was a $6 \%$ to $10 \%$ drop in birth weights of the infants who were in utero during the famine. There was no reduction in IQ at military induction, suggesting that fetal exposure to the famine did not cause mental retardation.

Although I had been aware of Pembrey's discussion ${ }^{17}$ of the famine in the context of what he calls transgenerational effects on fetal growth, I only recently discovered that the Dutch famine was also associated with an aberration in the incidence of schizophrenia. ${ }^{18}$ Not surprisingly, based on our current knowledge of neural tube defects and folic acid, there was a relative risk of $2.5(95 \%$ confidence interval $=1.3-4.9)$ for neural tube defects in the offspring of the cohort of women starved during first trimester. There was a remarkably parallel increase in the relative risk of schizophrenia to 2.0 (95\% confidence interval $=1.0-3.7)$. The authors describing the change in incidence of schizophrenia suggest that periconceptional folate deficiency may play a role in the etiology of schizophrenia. ${ }^{18}$ They observed a remarkable parallel in the relative risk for neural tube defects, schizophrenia, and schizophrenia spectrum personality disorders for first-trimester exposure to famine.

The impact of folic acid intake on DNA methylation has not been studied extensively, but there is some evidence for potentially important effects. The agouti viable yellow allele in mice is reported to be modulated by imprinting, strain-specific modification, and maternal epigenetic inheritance. Wolff et al. ${ }^{19}$ showed that expression could also be modified by maternal methyl-supplemented diet (choline, betaine, folate, $\mathrm{B}_{12}$, methionine, zinc). Whitelaw and colleagues ${ }^{20}$ have reported that the epigenotype for the agouti viable yellow alleles can be inherited in a non-Mendelian fashion. There is limited information regarding the effect of folic acid intake on DNA methylation in humans, but one study suggests that folic acid supplementation increases global DNA methylation in postmenopausal women. ${ }^{21}$ Studies evaluating the chemopreventive effect of folic acid in colon cancer have also provided some evidence for increased DNA methylation in response to folate supplementation. ${ }^{22}$ We have even less information about the effects of diet on methylation of histones. I would argue that there is an urgent need for more data regarding the general potential for modifying epigenotype through diet. We should attempt to determine whether folate supplementation reduces the risk of neural tube defects through methylation of DNA, histone, lipid, amino acid, protein, or other metabolite, and if it reduces the risk of schizophrenia. We urgently need to know whether preconceptional, prenatal, and postnatal folate supplementation is affecting methylation of DNA or histones and decreasing or increasing the incidence of epigenetic diseases. 


\section{References}

1. Tycko B, Ashkenas J. Epigenetics and its role in disease. J Clin Invest 2000;105:245246.

2. Petronis A. Human morbid genetics revisited: relevance of epigenetics. Trends Genet 2001;17:142-146.

3. Spence JE, Perciaccante RG, Greig GM, Willard HF, Ledbetter DH, Hejtmancik JF, Pollack MS, O’Brien WE, Beaudet AL. Uniparental disomy as a mechanism for human genetic disease. Am J Hum Genet 1988;42:217-226.

4. Wu C, Morris JR. Genes, genetics, and epigenetics: a correspondence. Science 2001; 293:1103-1105.

5. Holliday R. DNA methylation in eukaryotes: 20 years on. In: Russo V, Martienssen RA, Riggs AD, editors. Epigenetic mechanisms of gene regulation. Cold Spring Harbor, NY: Cold Spring Harbor Press, 2002:5-27.

6. Beck S, Olek A, Walter J. From genomics to epigenomics: a loftier view of life. Nat Biotechnol 1999;17:1144.

7. Jenuwein T, Allis CD. Translating the histone code. Science 2001;293:1074-1080.

8. Grunau C, Clark SJ, Rosenthal A. Bisulfite genomic sequencing: systematic investigation of critical experimental parameters. Nucleic Acids Res 2001;29:E65.

9. Petronis A, Gottesman II, Crow TJ, DeLisi LE, Klar AJ, Macciardi F, McInnis MG, McMahon FJ, Paterson AD, Skuse D, Sutherland GR. Psychiatric epigenetics: a new focus for the new century. Mol Psychiatry 2000;5:342-346.

10. Cook EH Jr, Lindgren V, Leventhal BL, Courchesne R, Lincoln A, Shulman C, Lord C, Courchesne E. Autism or atypical autism in maternally but not paternally derived proximal 15q duplication. Am J Hum Genet 1997;60:928-934.

11. Martinsson T, Johannesson T, Vujic M, Sjostedt A, Steffenburg S, Gillberg C, Wahlstrom J. Maternal origin of inv dup (15) chromosomes in infantile autism. Eur Child Adolesc Psychiatry 1996;5:185-192.
12. Wolpert CM, Menold MM, Bass MP, Qumsiyeh MB, Donnelly SL, Ravan SA, Vance JM, Gilbert JR, Abramson RK, Wright HH, Cuccaro ML, Pericak-Vance MA. Three probands with autistic disorder and isodicentric chromosome 15. Am J Med Genet 2000;96:365-372.

13. Borgatti R, Piccinelli P, Passoni D, Dalpra L, Miozzo M, Micheli R, Gagliardi C, Balottin U. Relationship between clinical and genetic features in "inverted duplicated chromosome 15" patients. Pediatr Neurol 2001;24:111-116.

14. Lauritsen M, Ewald H. The genetics of autism. Acta Psychiatr Scand 2001;103:411427.

15. Lumey LH, Ravelli AC, Wiessing LG, Koppe JG, Treffers PE, Stein ZA. The Dutch Famine Birth Cohort Study: design, validation of exposure, and selected characteristics of subjects after 43 years follow-up. Paediatr Perinat Epidemiol 1993;7:354-367.

16. Stein $\mathrm{Z}$ et al. Famine and human development: the Dutch hunger winter of 19441945. New York: Oxford University Press, 1975.

17. Pembrey M. Imprinting and transgenerational modulation of gene expression: human growth as a model. Acta Genet Med Gemellol (Roma) 1996;45:111-125.

18. Hoek HW, Brown AS, Susser E. The Dutch famine and schizophrenia spectrum disorders. Soc Psychiatry Psychiatr Epidemiol 1998;33:373-379.

19. Wolff GL, Kodell RL, Moore SR, Cooney CA. Maternal epigenetics and methyl supplements affect agouti gene expression in Avy/a mice. FASEB J 1998;12:949-957.

20. Morgan HD, Sutherland HG, Martin DI, Whitelaw E. Epigenetic inheritance at the agouti locus in the mouse. Nat Genet 1999;23:314-318.

21. Rampersaud GC, Kauwell GP, Hutson AD, Cerda JJ, Bailey LB. Genomic DNA methylation decreases in response to moderate folate depletion in elderly women. Am J Clin Nutr 2000;72:998-1003.

22. Kim YI, Baik HW, Fawaz K, Knox T, Lee YM, Norton R, Libby E, Mason JB. Effects of folate supplementation on two provisional molecular markers of colon cancer: a prospective, randomized trial. Am J Gastroenterol 2001;96:184-195. 